\title{
Prevalence and Risk Factors for Chronic Kidney Disease in Family Relatives of a Cameroonian Population of Hemodialysis Patients: A Cross- Sectional Study
}

\author{
Mazou Ngou Temgoua ${ }^{1,2}$, Gloria Ashuntantang1, Marie José Essi² ${ }^{2}$ Joël Nouktadie Tochie ${ }^{3 *}$, Moussa \\ Oumarou $^{4}$, Acho Fon Abongwa ${ }^{3}$, Aimé Mbonda ${ }^{2}$, Samuel Kingue ${ }^{1}$ \\ 'Department of Internal Medicine, Faculty of Medicine and Biomedical Sciences, University of Yaoundé 1, Yaoundé, \\ Cameroon \\ ${ }^{2}$ Department of Public Health, Faculty of Medicine and Biomedical Sciences, University of Yaoundé 1, Yaoundé, \\ Cameroon \\ ${ }^{3}$ Faculty of Medicine and Biomedical Sciences, University of Yaoundé 1, Yaoundé, Cameroon \\ ${ }^{4}$ Department of Internal Medicine, Douala General Hospital, Douala, Cameroon
}

*Corresponding Author: Joël Nouktadie Tochie, M.D., Faculty of Medicine and Biomedical Sciences, University of Yaoundé 1, Yaoundé, Cameroon. Tel: +237-676558825, Email: joeltochie@gmail.com

Received June 27, 2018; Accepted October 21, 2018; Online Published January 26, 2019

\begin{abstract}
Background: In sub-Saharan Africa (SSA), the trend in the number of patients admitted for maintenance hemodialysis is on the rise. The identification of risk factors for chronic kidney disease (CKD) ensures adequate primary and secondary preventive measures geared at reducing the burden of CKD in low-resource settings. A family history of CKD is an established risk factor for CKD in high-income countries. However, data on family predisposition to CKD is scarce in the literature on SSA.

Objective: The current study aimed to determine the prevalence and risk factors of CKD in family relatives of a Cameroonian population of hemodialysis patients (HDP) followed-up in a major hemodialysis referral center in Cameroon.

Methods: The current cross-sectional study was conducted over four months on a consecutive sample of first-degree family relatives of end-stage renal disease patients undergoing maintenance hemodialysis at the hemodialysis unit of the General Hospital of Yaoundé. For each participating family relative, socio-demographic characteristics, clinical data, and biological data including fasting blood glucose, proteinuria, and serum creatinine were collected.

Results: A total of 82 first-degree family relatives of HDP were recruited. The prevalence of CKD among the participants was $15.8 \%$. The main identified risk factors for CKD were age $(P=0.0015)$, female gender $(P=0.0357)$, hypertension $(P=$ $0.0004)$, regular intake of herbal remedies $(P=0.0214)$, and diabetes mellitus $(P=0.0019)$.

Conclusion: Overall, the current findings suggest an urgent need for population education, routine screening of CKD, and the identification of risk factors in first-degree family relatives of HDP in Cameroon.

Keywords: Chronic Kidney Disease, Prevalence, Risk Factors, Hemodialysis
\end{abstract}

\section{Background}

Chronic kidney disease (CKD) is an emerging global public health problem with an annual prevalence rate of $6 \%$ and affects $11 \%$ to $13 \%$ of the world's population. ${ }^{1,2}$ The economic burden of CKD is quite alarming; the costs of its management were evaluated at US\$16.74 and US $\$ 42.5$ billion in 1998 and 2009, respectively., Likewise, the global number of CKD-related deaths increased tremendously from 10478 deaths in 1980 to 90118 deaths in 2009., ${ }^{3,4}$ Though there is paucity of epidemiological data on CKD from sub-Saharan Africa (SSA), available hospital-based studies clearly depict that the rising incidence of hypertension and diabetes parallels the trend in the number of patients admitted for maintenance hemodialysis therapy. ${ }^{5}$ Similarly, between the year 2000 and 2004, the number of hemodialysis patients (HDP) for CKD in Cameroon doubled from 30 to 75 , corresponding to a $150 \%$ increase rate. ${ }^{6}$ In these resource-constraint settings, renal transplantation systems are nonexistent, and only 50 patients per million population are able to benefit from maintenance hemodialysis therapy. ${ }^{6}$ Despite recent government subsidization of the price of hemodialysis

Copyright $\odot 2019$ The Author(s). This is an open-access article distributed under the terms of the Creative Commons Attribution License (http:// creativecommons.org/licenses/by/4.0), which permits unrestricted use, distribution, and reproduction in any medium, provided the original work is properly cited. 
sessions in Cameroon, adherence to maintenance hemodialysis by CKD patients still remains a considerable concern. ${ }^{\text {? }}$

Because maintenance hemodialysis is considered a terminal therapeutic measure with high morbidity and mortality, ${ }^{8,9}$ identifying the risk factors of $\mathrm{CKD}$ is an important step toward determining primary preventive measures geared at reducing the incidence of CKD and secondary preventive measures aimed at curbing the number of CKD patients from progressing to end-stage renal disease where renal replacement therapy is the ultimate treatment for survival. ${ }^{10}$ Well-established risk factors for CKD are advanced age $>60$ years, hypertension, diabetes mellitus, and a family history of CKD. ${ }^{11}$ Current guidelines for the management of CKD do not stipulate routine screening for family relatives of CKD patients. ${ }^{12}$ Although reports on the prevalence and risk factors for CKD in family relatives of affected patients exist elsewhere, ${ }^{13-16}$ such invaluable data is scarce in SSA where other potential contributing factors like chronic infections (e.g., HIV/AIDS, ${ }^{17}$ hepatitis $\mathrm{B}$ and $\mathrm{C}$ infections ${ }^{18}$ ) and herbal remedies abuse are very frequent and likely to aggravate the burden of CKD. ${ }^{19-21}$

\section{Objective}

This study aimed to determine the prevalence and risk factors of CKD in the family relatives of a Cameroonian population of HDP followed-up in a major hemodialysis referral center in the capital city of Cameroon between December 2013 and April 2014.

\section{Methods}

This was a hospital-based, prospective, cross-sectional study enrolling all consecutive consenting parents, siblings, and children of HDP followed up at the hemodialysis unit of the General Hospital (YGH) of Yaoundé from December 15, 2013 to April 15, 2014. The YGH is a tertiary care center and university teaching hospital located in Yaoundé, the political capital of Cameroon. The hemodialysis unit of $\mathrm{YGH}$ is a major referral center for the investigation and management of end-stage renal disease in Yaoundé and its environs. Family relatives younger than 18 years of age, those pregnant, and those with hereditary kidney diseases like polycystic kidney disease were excluded. The minimal sample size was determined assuming an annual prevalence rate of CKD of $6 \%^{1}$ and a precision of $5 \%{ }^{22}$; hence, a minimum of 56 first-degree family relatives of HDP were required as study participants.

Through researcher administration of questionnaires, physical examinations, and standard laboratory procedures, the following variables from each consenting participant were studied:

- Socio-demographic characteristics (age and gender).

- Behavioral factors: the regular intake (at least once per week for not less than three months) of non-steroidal anti-inflammatory drugs (NSAIDs) and herbal remedies.
- Known co-morbidities: hypertension, diabetes mellitus, obesity

- Signs and symptoms of CKD: fatigue, nausea, vomiting, nocturia, facial and lower limb edema.

- Blood pressure: measured using standardized methods with the aid of an electronic sphygmomanometer $\left(\mathrm{Omron}^{\circledR}\right)$ and repeated 24 hours after the first measurement when results were above $140 / 90 \mathrm{~mm}$ $\mathrm{Hg}$.

- Fasting blood glucose: measured using a OneTouch Ultra $2^{\circledR}$ glucometer and repeated 24 hours after when values surpassed $126 \mathrm{mg} / \mathrm{dL}$.

- Proteinuria: measured in the first morning urine sample using a Cypress ${ }^{\circledR}$ dipstick. When the dipstick detected proteinuria, urinalysis was repeated three months later to confirm chronicity. ${ }^{12}$ The 24-hour urinary protein was not measured due to limited laboratory facilities in the resource-constrained environment.

- Serum creatinine: measured by the Jaffe method using a Roche-Hitachi Cobas $\mathrm{C} 311^{\circledR}$ analyzer. When the serum creatinine level was above $13 \mathrm{mg} / \mathrm{L}$, the test was repeated one week and three months later to confirm CKD. Electrolytes, urea, and uric acid were not measured due to limited laboratory facilities in the resource-challenged setting.

Definition of terms: CKD was defined as the persistence of proteinuria for more than three months and estimated glomerular filtration rate $(\mathrm{eGFR})^{12}<60 \mathrm{~mL} / \mathrm{min} / 1.73 \mathrm{~m}^{2}$ or when the disease was already diagnosed by nephrologists. Proteinuria was considered present if there was at least one cross on the dipstick. The eGFR was calculated using the Modification of Diet in Renal Disease (MDRD) formula ${ }^{23}$ as eGFR $=186 \times$ [serum creatinine $\left.(\mathrm{mg} / \mathrm{L})\right]^{-1,154}$ $\mathrm{x}$ [age (years) $]^{-0,203} \mathrm{x}$ (0.742 in female) $\mathrm{x} 0.192$. Diabetes mellitus was defined as a fasting blood glycemia $\geq 1.26 \mathrm{~g} /$ $\mathrm{L}^{24}$ or the patient having a past history of diabetes mellitus. Hypertension was defined as a systolic blood pressure $\geq 140 \mathrm{~mm} \mathrm{Hg}$ and/or diastolic blood pressure $\geq 90 \mathrm{~mm}$ $\mathrm{Hg}$ following two successive measurements at a 24 -hour interval or if the patient was known to be hypertensive. ${ }^{25}$ Obesity was considered as a body mass index (weight divided by in height squared) $\geq 30 \mathrm{~kg} / \mathrm{m}^{2} .^{26}$ First-degree family relatives were parents, siblings, and children of the HDP.

Data was analyzed using Statistical Package of Social Sciences (SPSS) Software, version 18. All variables were distributed in the form of simple frequencies, and means of numerical variables were reported. The threshold for statistical significance was set at 0.05 . Patients unavailable for follow-up with further laboratory tests were excluded from the final analysis.

\section{Results}

A total number of 82 family relatives corresponding to 35 (43\%) siblings, 35 (43\%) children, and 12 (14\%) parents of HDP agreed to participate in the current study. The mean 
age of the participants was $38.39 \pm 14.85$ years. The female gender was predominant with $66(72 \%)$ participants.

Out of the 82 family relatives of HDP, 13 were diagnosed with $\mathrm{CKD}$, indicating a prevalence of $15.85 \%$ which was significantly higher in parents compared to siblings and children (See Table 1). Among the 13 diagnosed cases of CKD, seven were newly diagnosed based on serum creatinine and/or proteinuria levels, while six had already been diagnosed with $\mathrm{CKD}$ by a nephrologist prior to enrollment into this study. The risk factors associated with CKD (persistence of proteinuria for more than three months and eGFR $<60 \mathrm{~mL} / \mathrm{min} / 1.73 \mathrm{~m}^{2}$ or when the disease was already diagnosed by nephrologists, as defined in the methods above) were having an age between 31 and 50 years, female gender, hypertension, regular intake of herbal remedies, and diabetes mellitus (all $P<0.05$ ) (Tables 1,2 , and 3).

\section{Discussion}

The present study aimed to determine the prevalence of $\mathrm{CKD}$ and its risk factors in the first-degree family relatives of a self-selected sub-Saharan African population of HDP receiving care at a major hemodialysis referral center in the capital city of Cameroon. The study found that the prevalence of CKD among the participants was $15.85 \%$ and more significant in parents. Being aged between 31 and 50 years, being of the female gender, having hypertension and/or diabetes mellitus, and regularly consuming herbal remedies were all risk factors for $\mathrm{CKD}$ in first-degree family relatives of HDP.

The prevalence of CKD in family relatives of HDP was slightly higher than the global prevalence of CKD which is estimated to be between $11 \%$ and $13 \% .^{1,2}$ The prevalence rate in the current study (15.85\%) was analogous to prevalence rates varying between $14.6 \%$ and $15.8 \%$ observed in Asia. ${ }^{20,21}$ This finding showed that relatives of CKD patients are at increased risk of CKD possibly because of a genetic link or environmental exposure.

As expected, CKD in family relatives of HDP was associated with advanced age. This finding concurs with previous reports which have indicated that eGFR deteriorates simultaneously with aging. ${ }^{27,28}$ For instance, the prevalence of CKD in the Chinese general population increases from $7.4 \%$ among those aged between 18-39 years to $18.0 \%$ and $24.2 \%$ among those aged $60-69$ and 70 years, respectively. ${ }^{29}$

Table 1. Prevalence of CKD, Laboratory Parameters, and Sociodemographic Characteristics Associated With CKD in First-Degree Family Relatives of Hemodialysis Patients

\begin{tabular}{|c|c|c|c|c|c|}
\hline Groups & $\begin{array}{l}\text { Number }(\%) \\
\quad(\mathbf{n}=82)\end{array}$ & $\begin{array}{c}\text { Parents } \\
\mathrm{n}=12(\%)\end{array}$ & $\begin{array}{c}\text { Siblings } \\
\mathrm{n}=35(\%)\end{array}$ & $\begin{array}{c}\text { Children } \\
n=35(\%)\end{array}$ & $P$ value \\
\hline Proportions of CKD & 13 (15.85). & $7(58.3)$ & $4(11.4)$ & $2(5.7)$ & $<0.0001$ \\
\hline \multicolumn{6}{|l|}{ Age (years) } \\
\hline $18-30$ & $21(25.6)$ & $01(8.3)$ & $06(17.1)$ & $14(40)$ & \\
\hline $31-50$ & $41(50)$ & $06(50)$ & $14(40)$ & $21(60)$ & 0.0015 \\
\hline $51-70$ & $16(19.5)$ & $04(33.3)$ & $12(34.3)$ & $0(0)$ & \\
\hline$>70$ & $4(4.8)$ & $01(8.3)$ & $03(8.6)$ & $0(0)$ & \\
\hline \multicolumn{6}{|l|}{ Gender } \\
\hline Male & $16(28)$ & 05 (41.7) & 08 (22.9) & $03(8.6)$ & 0.0357 \\
\hline Female & $66(72)$ & $07(58.3)$ & $27(77.1)$ & $32(91.4)$ & \\
\hline Serum creatinine (mean in mg/L) & $9.21 \pm 6.6$ & & & & \\
\hline Fasting blood glucose (mean in mg/dL) & $92 \pm 31$ & & & & \\
\hline Proteinuria (mean in mg) & $468 \pm 210$ & & & & \\
\hline
\end{tabular}

Abbreviation: CKD, chronic kidney disease.

Table 2. Behavioral Factors Associated With CKD in First-Degree Family Relatives of Hemodialysis Patients

\begin{tabular}{|c|c|c|c|c|c|}
\hline Groups & $\begin{array}{c}\text { Number }(\%) \\
(n=82)\end{array}$ & $\begin{array}{c}\text { Parents } n=12 \\
(\%)\end{array}$ & $\begin{array}{c}\text { Siblings } \mathrm{n}=35 \\
(\%)\end{array}$ & Children $n=35(\%)$ & $P$ Value \\
\hline Regular intake of herbal remedies & $19(23.2)$ & $06(50)$ & $09(25.7)$ & $04(11.4)$ & 0.0214 \\
\hline Regular intake of NSAIDs & $14(17.1)$ & $03(25)$ & $06(17.1)$ & $05(14.3)$ & 0.6960 \\
\hline
\end{tabular}

Abbreviations: CKD, chronic kidney disease; NSAIDS, non-steroidal anti-inflammatory drugs.

Table 3. Co-morbidities Associated With CKD in First-Degree Family Relatives of Hemodialysis Patients

\begin{tabular}{|c|c|c|c|c|c|}
\hline Groups & $\begin{array}{c}\text { Number }(\%) \\
(n=82)\end{array}$ & $\begin{array}{c}\text { Parents } \\
n=12(\%)\end{array}$ & $\begin{array}{c}\text { Siblings } \\
n=35(\%)\end{array}$ & $\begin{array}{c}\text { Children } \\
n=35(\%)\end{array}$ & $P$ Value \\
\hline Hypertension & $26(31.7)$ & $7(58.3)$ & $16(45.7)$ & $3(8.6)$ & 0.0004 \\
\hline Obesity & $12(14.6)$ & $2(16.7)$ & $7(20)$ & $3(8.6)$ & 0.3914 \\
\hline Diabetes mellitus & $4(4.9)$ & $3(25)$ & $0(0)$ & $1(2.9)$ & 0.0019 \\
\hline
\end{tabular}

Abbreviation: CKD, chronic kidney disease. 
Generally, women have a longer life span than men, ${ }^{30}$ and this may explain the association between CKD and the female gender in the current study. In contrast, reports by Silbiger and Neugarten ${ }^{31}$ demonstrated a more rapid decline in kidney function in males compared to females after controlling confounders like hypertension or dylipidemia. Additionally, contrasting evidence suggests that female sex hormones like estrogen may exert potent antioxidant actions on mesangial cells, which may contribute to the protective effect of the female gender against CKD. ${ }^{31}$

Having hypertension emerged as a risk factor of CKD, probably explained by the effects of sustained uncontrolled high blood pressure on the kidneys which hastens the progression of CKD. ${ }^{32,33}$ The proportion of hypertension $(31.7 \%)$ in the family relatives of HDP was similar to the national prevalence $(31 \%)$ of hypertension in Cameroon. ${ }^{34}$ Inserra et al in Argentina found a relatively higher hypertension prevalence of $41.8 \%$ in family relatives of HDP $^{35}$ This is probably because their method of blood pressure measurement was not standardized, but based on readings of a single point in time compared to the serial confirmatory readings used in our methods. Therefore, it is likely that the prevalence of hypertension they obtained was overestimated.

Likewise, having diabetes mellitus was a significant risk factor for CKD in family relatives of HDP patients, probably explained by the role played by diabetic nephropathy in the pathogenesis of CKD. ${ }^{36}$ The prevalence of diabetes in family relatives of HDP was $4.9 \%$ and consistent with the $5.2 \%$ prevalence rate obtained by Inserra et al in family relatives of HDP of Argentina. ${ }^{35}$ However, the current finding was inferior to the prevalence of diabetes $(6.1 \%)$ reported in the general population of Cameroon in the year 2010. ${ }^{37}$ The relatively low prevalence of diabetes in the current study can be attributed to selection bias; the young study population with a mean age of 38 years was unlikely to have diabetes as opposed to more elderly study participants.

Similarly to Vivekanand ${ }^{38}$ and $\mathrm{Hsieh}$ et $\mathrm{al}^{39}$ an association between CKD and the ingestion of herbal medicine was found in the current study. Nephrotoxicity leading to $\mathrm{CKD}$ has been reported in association with several herbs, especially with the Aristolochia plant. ${ }^{38}$ In the current study, the exact herbs ingested could not be precisely identified by participants. It is noteworthy that the frequency of regular intake of herbal remedies $(23.2 \%)$ in the present study largely exceeded the $2.6 \%$ and $6.4 \%$ reported by Tsai et al in Taiwan ${ }^{20}$ and Kong et al in China. ${ }^{21}$ Possible explanations for this discrepancy include the lack of awareness of the potential nephrotoxic effects of these substances on the part of family relatives of HDP. The current findings reiterate previous reports of the World Health Organization which highlighted the consumption of traditional remedies as an important health concern in Africa. ${ }^{40}$

We acknowledge the following potential limitations of our study:
- Its suboptimal sample size $(n=82)$ which renders the results generalizable to the entire Cameroonian population of family relatives of HDP with caution.

- The absence of a control group to bring forth statistically significant risk factors for CKD in family relatives of HDP.

Also, this study was not designed to identify genes predisposing family relatives of HDP to CKD, highlighting the need for further research in this domain. However, following an extensive literature search, to the best of our knowledge, this is one of the first studies highlighting the relation between CKD and family predisposition in Cameroon. Its additional merits include its robust methods using standardized clinical and laboratory procedures.

\section{Conclusion}

The current study is one of the first to highlight the relation between CKD and family predisposition in Cameroon and perhaps SSA at large. Its findings suggest that at least one out of every ten first-degree family relatives of HDP have CKD. The risk factors for CKD were being aged between 31 and

\section{Research Highlights}

\section{What Is Already Known?}

In sub-Saharan Africa, the trend in the number of patients admitted for maintenance hemodialysis is on the rise. The identification of risk factors for CKD ensures the development of adequate primary and secondary preventive measures geared toward reducing the burden of CKD in these low-resource settings. Family history of $\mathrm{CKD}$ is an established risk factor for CKD in high-income countries. However, data on family predisposition to $\mathrm{CKD}$ is scarce in the literature on sub-Saharan Africa.

\section{What This Study Adds?}

This study aimed to contribute to the current scarcity of data on the relationship between CKD and family predisposition in sub-Saharan Africa. We have conducted one of the first studies highlighting the relation between having CKD and family predisposition in Cameroon and perhaps sub-Saharan Africa at large. The current findings suggest that at least one out of every ten first-degree family relatives of HDP have CKD. The main identified risk factors for CKD were age, female gender, hypertension, regular intake of herbal remedies, and diabetes. These findings are of great public health importance, as the results suggest an urgent need for population education on the above risk factors for CKD. Moreover, the study is innovative in that it provides preliminary data which may warrant routine screening for CKD and the above risk factors in first-degree family relatives of HDP in Cameroon. With the increasing morbidity and mortality of CKD in sub-Saharan Africa, such preventive measures cannot be overemphasized. 
50 years, being of the female gender, having hypertension, regularly consuming herbal remedies, and having diabetes. Overall, the current findings suggest an urgent need for population education and routine screening for CKD and the identified risk factors in the first-degree family relatives of HDP in Cameroon.

\section{Authors' Contributions}

MNT, GA, MJE, and SK: study conception and design, data collection and analysis, interpretation of results and critical revisions. JNT, MO, AM, and AFA: data analysis, interpretation of results, manuscript writing, and critical revision. All the authors read and approved the final version of the manuscript.

\section{Conflict of Interest Disclosures}

The authors declare that they have no conflicts of interest.

\section{Ethical Approval}

Ethical approval was obtained from the Institutional Review Board of the Faculty of Medicine and Biomedical Sciences, University of Yaoundé I prior to the start of the study under No. IEC-UYI/454/02/2014/N. Administrative authorization was obtained from the Director of the General Hospital of Yaoundé prior to the start of the study. Written informed consent was obtained from all participants who took part in this study.

\section{Acknowledgments}

The authors gratefully acknowledge the study participants for their commitment and equally thank the administrative authorities of Yaoundé General Hospital for granting the authorization to carry out this study.

\section{References}

1. Hill NR, Fatoba ST, Oke JL, et al. Global Prevalence of Chronic Kidney Disease - A Systematic Review and Meta-Analysis. PLoS One. 2016;11(7):e0158765. doi:10.1371/journal. pone.0158765.

2. Baumgarten M, Gehr T. Chronic kidney disease: detection and evaluation. Am Fam Physician. 2011;84(10):1138-1148.

3. Bello AK, Nwankwo E, El Nahas AM. Prevention of chronic kidney disease: a global challenge. Kidney Int Suppl. 2005(98):S11-17. doi:10.1111/j.1523-1755.2005.09802.x.

4. National Kidney and Urologic Diseases Information Clearinghouse (NKUDIC)/National Institutes of Health (NIH). Kidney disease statistics for the United States. NIH Publication; 2012.

5. Bakris GL, Ritz E. The message for World Kidney Day 2009: hypertension and kidney disease--a marriage that should be prevented. J Hypertens. 2009;27(3):666-669. doi:10.1097/ HJH.0b013e328327706a.

6. El Matri A, EAM Elhassan, Abu-Aisha H. Renal replacement therapy resources in Africa. Arab J Nephrol Transplant. 2008;1(1):9-14. doi:10.4314/ajnt.v1i1.58814.

7. Mosima E. Cameroon: "More Dialysis Centres Have Facilitated Treatment". allAfrica.com website. http://allafrica.com/ stories/201601060720.html. Accessed June 30, 2018.

8. Ozer Etik D, Ocal S, Boyacioglu AS. Hepatitis C infection in hemodialysis patients: A review. World J Hepatol. 2015;7(6):885-895. doi:10.4254/wjh.v7.i6.885.
9. Khan $\mathrm{YH}$, Sarriff A, Adnan AS, Khan AH, Mallhi TH. Blood Pressure and Mortality in Hemodialysis Patients: A Systematic Review of an Ongoing Debate. Ther Apher Dial. 2016;20(5):453-461. doi:10.1111/1744-9987.12406.

10. Definition, Classification, and Prognosis in CKD. KDIGO. http://kdigo.org/home/conferences/definition-classificationand-prognosis-in-ckd-2009/. Accessed June 30, 2018.

11. Levey AS, Atkins R, Coresh J, et al. Chronic kidney disease as a global public health problem: approaches and initiatives a position statement from Kidney Disease Improving Global Outcomes. Kidney Int. 2007;72(3):247-259. doi:10.1038/ sj.ki.5002343.

12. Levin A, Stevens PE, Bilous RW, et al. Kidney disease: Improving global outcomes (KDIGO) CKD work group. KDIGO 2012 clinical practice guideline for the evaluation and management of chronic kidney disease. Kidney Int Suppl. 2013;3(1):1-150. doi:10.1038/kisup.2012.73.

13. Bello AK, Peters J, Wight J, de Zeeuw D, El Nahas M. A population-based screening for microalbuminuria among relatives of CKD patients: the Kidney Evaluation and Awareness Program in Sheffield (KEAPS). Am J Kidney Dis. 2008;52(3):434443. doi:10.1053/j.ajkd.2007.12.034.

14. Bergman S, Key BO, Kirk KA, Warnock DG, Rostant SG. Kidney disease in the first-degree relatives of African-Americans with hypertensive end-stage renal disease. Am J Kidney Dis. 1996;27(3):341-346. doi:10.1016/S0272-6386(96)90356-X.

15. Brown WW, Peters RM, Ohmit SE, et al. Early detection of kidney disease in community settings: the Kidney Early Evaluation Program (KEEP). Am J Kidney Dis. 2003;42(1):2235. doi:10.1016/S0272-6386(03)00405-0.

16. O'Dea DF, Murphy SW, Hefferton D, Parfrey PS. Higher risk for renal failure in first-degree relatives of white patients with endstage renal disease: a population-based study. Am J Kidney Dis. 1998;32(5):794-801.doi:10.1016/S0272-6386(98)70135-0.

17. Tianyi FL, Tochie JN, Agbor VN, Kadia BM. Audit of HIV counselling and testing services among primary healthcare facilities in Cameroon: a protocol for a multicentre national cross-sectional study. BMJ Open. 2018;8(3):e020611. doi:10.1136/bmjopen-2017-020611.

18. Njigou AR, Tochie JN, Danwang C, et al. Seroprevalence of Hepatitis B and C Infections in Mokolo District Hospital, Northern Cameroon: The Value of a Screening Campaign. Hosp Pract Res. 2018;3(3):79-84. doi:10.15171/hpr.2018.18.

19. Ayodele OE, Salako BL, Kadiri S, Arije A, Alebiosu CO. Hepatitis B virus infection: implications in chronic kidney disease, dialysis and transplantation. Afr J Med Med Sci. 2006;35(2):111-119.

20. Stanifer JW, Jing B, Tolan S, et al. The epidemiology of chronic kidney disease in sub-Saharan Africa: a systematic review and meta-analysis. Lancet Glob Health. 2014;2(3):e174-181. doi:10.1016/s2214-109x(14)70002-6.

21. Stanifer JW, Lunyera J, Boyd D, et al. Traditional medicine practices among community members with chronic kidney disease in northern Tanzania: an ethnomedical survey. BMC Nephrol. 2015;16:170. doi:10.1186/s12882-015-0161-y.

22. Eng J. Sample size estimation: how many individuals should be studied? Radiology. 2003;227(2):309-313. doi:10.1148/ radiol.2272012051.

23. Estimating Glomerular Filtration Rate (GFR): National Institute of Diabetes and Digestive and Kidney Diseases (NIDDK). [cited 2018 Jun 30]. Available from: https://www.niddk.nih.gov/ health-information/health-communication-programs/nkdep/ lab-evaluation/gfr/estimating/Pages/estimating.aspx. Accessed June 30, 2018.

24. OMS. Diabète. http://www.who.int/mediacentre/factsheets/ fs312/fr/. Accessed June 30, 2018.

25. Arterial Hypertension (Management of). http://www.escardio. 
org/Guidelines/Clinical-Practice-Guidelines/ArterialHypertension-Management-of. Accessed June 30, 2018.

26. Reiner Z, Catapano AL, De Backer G, et al. ESC/EAS Guidelines for the management of dyslipidaemias: the Task Force for the management of dyslipidaemias of the European Society of Cardiology (ESC) and the European Atherosclerosis Society (EAS). Eur Heart J. 2011;32(14):1769-1818. doi:10.1093/ eurheartj/ehr158.

27. Tonelli M, Riella M. Chronic kidney disease and the aging population. Indian J Nephrol. 2014;24(2):71-74. doi:10.4103/0971-4065.127881.

28. O'Hare AM, Choi Al, Bertenthal D, et al. Age affects outcomes in chronic kidney disease. J Am Soc Nephrol. 2007;18(10):27582765.doi:10.1681/asn.2007040422.

29. Zhang L, Wang F, Wang L, et al. Prevalence of chronic kidney disease in China: a cross-sectional survey. Lancet. 2012;379(9818):815-822. doi:10.1016/s01406736(12)60033-6.

30. Iseki K. Gender differences in chronic kidney disease. Kidney Int. 2008;74(4):415-417. doi:10.1038/ki.2008.261.

31. Silbiger SR, Neugarten J. The impact of gender on the progression of chronic renal disease. Am J Kidney Dis. 1995;25(4):515-533. doi:10.1016/0272-6386(95)90119-1.

32. Judd E, Calhoun DA. Management of hypertension in CKD: beyond the guidelines. Adv Chronic Kidney Dis. 2015;22(2):116-122. doi:10.1053/j.ackd.2014.12.001.

33. Tedla FM, Brar A, Browne R, Brown C. Hypertension in chronic kidney disease: navigating the evidence. Int J Hypertens. 2011;2011:132405. doi:10.4061/2011/132405.

34. Kingue S, Ngoe CN, Menanga AP, et al. Prevalence and Risk Factors of Hypertension in Urban Areas of Cameroon: A Nationwide Population-Based Cross-Sectional Study. J Clin Hypertens (Greenwich). 2015;17(10):819-824. doi:10.1111/ jch.12604.

35. Inserra F, de la Llave $\mathrm{G}$, Alpino $\mathrm{M}$, et al. [Survey of risk factors and renal disease in first-degree relatives of dialysis patients]. Medicina (B Aires). 2007;67(1):8-18.

36. Cavanaugh KL. Diabetes management issues for patients with chronic kidney disease. Clin Diabetes. 2007;25(3):90-97. doi:10.2337/diaclin.25.3.90.

37. Mbanya JC, Motala AA, Sobngwi E, Assah FK, Enoru ST. Diabetes in sub-Saharan Africa. Lancet. 2010;375(9733):2254 2266. doi:10.1016/s0140-6736(10)60550-8.

38. Jha V. Herbal medicines and chronic kidney disease. Nephrology (Carlton). 2010;15 Suppl 2:10-17. doi:10.1111/ j.1440-1797.2010.01305.x.

39. Hsieh CF, Huang SL, Chen $\mathrm{CL}$, et al. Increased risk of chronic kidney disease among users of non-prescribed Chinese herbal medicine in Taiwan. Prev Med. 2012;55(2):155-159. doi:10.1016/j.ypmed.2012.06.003.

40. Regulation of Traditional Medicine in the WHO African Region. African Health Observatory. http://www.aho.afro.who. int/en/ahm/issue/13/reports/regulation-traditional-medicinewho-african-region. Accessed June 30, 2018. 